\title{
TRAUMA-INDUCED COAGULOPATHY: PROSPECTIVE OBSERVATION STUDY
}

\author{
Mariana Vyshynska
}

Coagulopathy remains the leading cause of illness and death in people with severe trauma.

The aim was to study the indicators of vascular-platelet hemostasis in severe trauma and to establish changes in the main pathophysiological mechanisms of primary hemostasis that occur in patients with trauma-induced coagulopathy, compared with almost healthy individuals of the same age.

Materials and methods. The study included 44 patients aged 19 to 55 years (36.1 [28.7; 43.2] years). The control group included 20 patients of the therapeutic department without preconditions for changes in the hemostasis system, and the main group - 24 patients with diagnosis of "severe trauma" who were treated in the anesthesiology and intensive care department.

Results and discussion. We studied indicators of intravascular platelet activation. Patients had a normal number of platelets in the venous blood, but the level of spherocytes and spheroechinocytes increased. On the 3rd day after the injury, number of platelets in the venous blood was normal, however the level of discocytes decreased, and the level of discochinocytes, spheroechinocytes and the sum of active forms of platelets increased. On the fifth day, the level of discochinocytes and active forms of platelets, significantly higher ( $p<0.05)$ among patients of the main group, and spherocytes, spheroechinocytes and platelets involved in the aggregates, were significantly lower than in the control group of patients $(p<0.05)$.

Conclusions. Indicators of vascular-platelet hemostasis in patients with polytrauma had significant differences from those of the control group. This may be evidence of activation of the vascular-platelet system of the hemostasis system and may be a prerequisite for late thrombotic complications in patients with polytrauma

Keywords: coagulopathy, polytrauma, coagulation hemostasis, vascular-platelet hemostasis, hypocoagulation, platelets, tranexamic acid

(C) The Author(s) 202

This is an open access article under the Creative Commons CC BY license

How to cite:

Vyshynska, M. (2021). Trauma-induced coagulopathy: prospective observation study. ScienceRise: Medical Science, 4 (43), 29-34. doi: http://doi.org/10.15587/2519-4798.2021.238094

\section{Introduction}

The main cause of morbidity and death in patients with polytrauma is coagulopathy induced by trauma [1]. It can be manifested by changes that are characteristic of both hypocoagulation and hypercoagulation. Early trauma-induced coagulopathy (which usually occurs within 6 hours after injury) is characterized by disturbances in hemostasis, which is the cause of severe bleeding and shock. Late (develops more than 24 hours after injury) is a consequence of hypercoagulation and leads to thromboembolic events (deep vein thrombosis, pulmonary embolism), as well as acute respiratory distress syndrome and multiple organ failure. Early and late coagulopathies due to trauma are not mutually exclusive. In addition, the transition from hypocoagulation to hypercoagulation can occur in a few minutes or in a few days [2].

In addition to the pool of coagulation factors, according to modern cellular theory of blood clotting, blood cells and, in particular, platelets play an important role in thrombus formation. In recent years, in scientific publications on trauma, many studies have focused on the study of changes in the actual platelet hemostasis [35]. In particular, it was found that platelet suppression occurs even with minor injuries [6]. It is difficult to assess the violation of platelet aggregation ex vivo, because there is no endothelium, blood flow, and, in fact, local tissue damage. However, even in such studies, it was found that with a normal platelet count, almost half of patients have disorders of platelet aggregation, but it is not studied to what extent these disorders are pathological. Careful assessment of platelet dysfunction is hampered by the technical complexity of existing techniques for studying platelet function under conditions that would meet the conditions of the human body. It is also unclear how to use and interpret vascular hemostasis in patients with trauma coagulopathy.

The aim of the research was to study the indicators of vascular-platelet hemostasis in polytrauma and to establish changes in the main pathophysiological mechanisms of primary hemostasis that occur in patients with coagulopathy due to trauma, compared with almost healthy individuals of the same age.

\section{Materials and methods}

The prospective observational study included 44 patients aged 19 to 55 years $(36.1$ [28.7; 43.2] years) 
who were treated at the Municipal Non-Profit Enterprise "8th City Clinical Hospital of Lviv" in the period from 01.01.2021 to 30.05.2021, the control group included 20 patients of the therapeutic department without preconditions for changes in the hemostasis system and in the absence of laboratory-confirmed disorders in the hemostasis system. The main group included 24 patients with a diagnosis of "polytrauma" who were treated in the anesthesiology department with intensive care.

Criteria for inclusion in the study were: consent of the patient or his legal representatives to participate in the study, polytrauma, the introduction of tranexamic acid in the first day after injury. Criteria for exclusion from the study were: the patient's refusal to participate in the study, medical history of congenital pathology of the hemostasis system, the agonal state of the patient.

The study was conducted in accordance with the requirements of the Helsinki Declaration of the World Medical Association, the Council of Europe Convention on Human Rights and Biomedicine, good clinical practice and approved by the Bioethics Commission of the 8th City Clinical Hospital in Lviv (Minutes No. 4 of 20.10.2020). Patients were included in the study after signing an informed consent. The severity of the patients' condition was assessed by the Injury Severity Score (ISS).

The main stages of the study were: prehospital stage (1st hour after injury), 1st, 3rd, 5th day after admission to treatment.

The following clinical markers were selected: platelet count and hematocrit; indicators of vascularplatelet hemostasis (intravascular platelet activation, platelet aggregation induced by adrenaline and ADP) were determined by turbinometric method, using a phase contrast microscope; indicators of coagulation hemostasis (prothrombin time, prothrombin index, INR (international normalized), APTT (activated partial thromboplastin time), total fibrinogen level) were determined quanti- tatively using diagnostic kits from "Siemens" on the semi-automatic coagulometer "SYSMEX 560".

Also we analyzed the volume of crystalloids and colloids at all stages of the study, the use of tranexamic acid in the prehospital stage and on the 1st day after admission to treatment, the volume of fresh frozen plasma and erythrocyte mass on the $1 \mathrm{st}, 3 \mathrm{rd}$, 5th that day from the moment of admission to treatment. The presence of disseminated intravascular coagulation syndrome was performed on the ISTH scale (International Society on Thrombosis and Haemostasis Score) using a medical calculator [7].

Treatment of patients with polytrauma was carried out in accordance with the Order of the Ministry of Health of Ukraine No. 1269 of 05.06.2019 "Emergency care: pre-hospital stage" (Chapter 8), "The European guideline on management of major bleeding and coagulopathy following trauma: fifth edition" (2019), and local protocols approved by the Municipal Non-Profit Enterprise "8th City Clinical Hospital of Lviv".

Statistical processing of the obtained data was performed using the statistical application package Microsoft Excel (2016). All obtained quantitative research results were subjected to statistical processing by methods of variation statistics using the values of arithmetic mean $(\mathrm{X})$, standard deviation $(\sigma)$, median $(\mathrm{Me})$, first and third quartiles [Q1; Q3], significance level (p). Under the condition of normal sample distribution, the data were processed using parametric criteria (Student's t-test), in case of non-compliance of the sample with the laws of normal distribution - non-parametric statistical methods (Mann-Whitney U-test). The difference in parameters was considered statistically significant at $\mathrm{p}<0.05$.

\section{Research results}

Anthropometric characteristics of patients who were included in the study are given in Table 1.

Table 1

Anthropometric characteristics of patients (Me [Q1; Q3])

\begin{tabular}{|c|c|c|}
\hline Parameters & Control group of patients $(\mathrm{n}=20)$ & Main group of patients $(\mathrm{n}=24)$ \\
\hline Age of patients & $38,4[29,1 ; 43,5]$ & $35,2[31,3 ; 44,8]$ \\
\hline Body weight, $\mathrm{kg}$ & $72,5[63,4 ; 81,9]$ & $74,3[68,2 ; 84,5]$ \\
\hline BMI, $\mathrm{kg} / \mathrm{m}^{2}$ & $25,9[19,8 ; 27,4]$ & $28,1[21,3 ; 30,1]$ \\
\hline
\end{tabular}

Note: $*-p<0.05$ compared with the control group of patients

We found no significant differences in age, body weight, body mass index (BMI) between patients in the control and main groups.

We assessed the severity of patients on the ISS scale (Table 2) and found that less than 9 points were not received by any of the patients of the main group, 9-15 points were evaluated by $20.8 \%$ of patients with polytrauma, $16-25$ points were $75 \%$ patients, more than 25 points $-4.2 \%$ of patients.

We conducted a study of intravascular platelet activation (Table 3) and found that on the first day, with a normal number of platelets in the venous blood, patients had increased levels of spherocytes and sphero echinocytes, and the level of intact platelets (discocytes) and disco echinocytes was within normal limits.
Table 2

Assessment of the severity of patients on a scale Injury Severity Score (ISS)

\begin{tabular}{|c|c|}
\hline ISS & The main group of patients $(\mathrm{n}, \%)$ \\
\hline$<9$ points & - \\
\hline $9-15$ points & $5(20.8 \%)$ \\
\hline $16-25$ points & $18(75 \%)$ \\
\hline$>25$ points & $1(4.2 \%)$ \\
\hline
\end{tabular}

The level of bipolar platelet forms, the sum of active platelet forms and the number of platelets involved in the aggregates were also within normal limits. On the 3rd day after the injury, the normal number of platelets in the venous blood also remained, but the level of discocytes decreased, and the level of disco echinocytes, sphe- 
ro echinocytes and the sum of active forms of platelets increased. The number of spherocytes and the number of platelets involved in the aggregates were within normal limits. Bipolar forms of platelets were not observed.

Table 3

Indicators of intravascular platelet activation at 1,3 and 5 days in patients with polytrauma $(\mathrm{X} \pm \sigma)$

\begin{tabular}{|c|c|c|c|c|c|}
\hline \multirow{2}{*}{\multicolumn{2}{|c|}{ Indicators }} & \multirow{2}{*}{$\begin{array}{l}\text { Control group of } \\
\text { patients } \\
(n=20)\end{array}$} & \multicolumn{3}{|c|}{ Main group of patients } \\
\hline & & & $\begin{array}{l}\text { 1st day }(\mathrm{d} 1) \\
(\mathrm{n}=24)\end{array}$ & $\begin{array}{l}\text { 3rd day }(\mathrm{d} 3) \\
\qquad(\mathrm{n}=24)\end{array}$ & $\begin{array}{l}5 \text { th day }(\mathrm{d} 5) \\
\qquad(\mathrm{n}=24)\end{array}$ \\
\hline \multicolumn{2}{|c|}{$\begin{array}{l}\text { The number of platelets in the ve- } \\
\text { nous blood, } 10^{9} / 1\end{array}$} & $202.7 \pm 34.7$ & $138.0 \pm 11.8 *$ & $176.0 \pm 23.2$ & $184.3 \pm 27.1$ \\
\hline \multirow{5}{*}{$\begin{array}{c}\text { Different } \\
\text { forms of } \\
\text { platelets, } \\
\%\end{array}$} & Discocytes & $82.6 \pm 1.6$ & $77.0 \pm 2.1 *$ & $81.5 \pm 1.6$ & $83,2 \pm 1,8$ \\
\hline & Disco echinocytes & $8.2 \pm 1.1$ & $18.0 \pm 1.9 *$ & $14.4 \pm 2.2 *$ & $12,1 \pm 2,4 *$ \\
\hline & Spherocytes & $5.2 \pm 0.7$ & $1.5 \pm 0.1 *$ & $2.5 \pm 0.2 *$ & $3,1 \pm 0,4 *$ \\
\hline & Sphero echinocytes & $3.1 \pm 0.4$ & $4.3 \pm 0.6$ & $2.6 \pm 0.2$ & $1,2 \pm 0,3 *$ \\
\hline & Bipolar forms & 0 & 0 & 0 & 0 \\
\hline \multicolumn{2}{|c|}{$\begin{array}{l}\text { The sum of the active forms of } \\
\text { platelets, } \%\end{array}$} & $12.9 \pm 2.7$ & $17.7 \pm 0.2 *$ & $21.2 \pm 1.6 *$ & $19.2 \pm 1.1 *$ \\
\hline \multicolumn{2}{|c|}{$\begin{array}{c}\text { The number of platelets involved in } \\
\text { the aggregates, } \%\end{array}$} & $14.1 \pm 3.4$ & $3.3 \pm 1.5 *$ & $6.3 \pm 0.2 *$ & $7.6 \pm 0.8 *$ \\
\hline
\end{tabular}

Note: $*-p<0.05$ compared with the control group of patients

On the fifth day after inclusion in the study, the number of platelets in the main group did not differ significantly from the control group, and no significant differences were found between the control and main groups in terms of discocyte levels and bipolar platelets. While the level of disco echinocytes and platelet active forms was significantly higher $(\mathrm{p}<0.05)$ among patients of the main group, and spherocytes, sphero echinocytes and platelets involved in aggregates were significantly lower than the control group of patients $(p<0.05)$. Thus, the results of indicators of intravascular platelet activation indicate the activation of the vascular-platelet link of hemostasis both on day 1 and on day 5 of the disease.

The study of coagulation hemostasis (Table 4) in patients with polytrauma on the 1 st day revealed a significant $(\mathrm{p}<0.05$ compared with the control group of patients) prolongation of prothrombin time, decrease in prothrombin index and total fibrinogen, increase in INR and APTT on the third day after receiving polytrauma in patients of the main group, all studied indicators, except for APTT and fibrinogen B, did not differ significantly from similar indicators of the control group, while on the fifth day - the level of total fibrinogen significantly $(p<0.05)$ exceeded indicators of the control group of patients.

The frequency of tranexamic acid administration at different time intervals after injury was analyzed (Fig.1). 4 patients out of 24 received $1 \mathrm{~g}$ of tranexamic acid in the prehospital stage, 18 patients - in the period from 3 to 8 hours, and 2 patients in the first day, but $>8$ hours. All 24 patients received a subsequent infusion of tranexamic acid at a dose of $1 \mathrm{~g}$ for 8 hours orally.

The frequency of transfusion of crystalloids and colloids on the 1st, 3rd and 5th days of treatment was analyzed (Fig. 2). It was found that on the 1st day of treatment the level of crystalloids and colloids was the highest (average dose $-20 \mathrm{ml} / \mathrm{kg}$ ), on the 5 th day - the lowest (average dose $-8 \mathrm{ml} / \mathrm{kg}$ ).

Table 4

Indicators of coagulation hemostasis in patients included in the study

\begin{tabular}{|c|c|c|c|c|}
\hline \multirow{2}{*}{ Indicators } & $\begin{array}{c}\text { Control group of } \\
\text { patients } \\
(\mathrm{n}=20)\end{array}$ & $\begin{array}{c}\text { Mst day }(\mathrm{d} 1) \\
(\mathrm{n}=24)\end{array}$ & $\begin{array}{c}\text { 3rd day }(\mathrm{d} 3) \\
(\mathrm{n}=24)\end{array}$ & $\begin{array}{c}5 \text { th day }(\mathrm{d} 5) \\
(\mathrm{n}=24)\end{array}$ \\
\cline { 3 - 5 } & $14.5 \pm 1.4$ & $21.4 \pm 2.1$ & $12.5 \pm 1.3$ & $14.9 \pm 2.2$ \\
\hline Prothrombin time, $\mathrm{s}$ & $84.2 \pm 5.2$ & $68.5 \pm 4.1 *$ & $78.1 \pm 2.5$ & $84.7 \pm 3.9$ \\
\hline Prothrombin index, \% & $1.12 \pm 0.15$ & $1.63 \pm 0.11 *$ & $1.39 \pm 0.09$ & $1.15 \pm 0.05$ \\
\hline INR & $28.5 \pm 3.4$ & $47.5 \pm 1.9 *$ & $42.1 \pm 2.5 *$ & $34.2 \pm 1.8$ \\
\hline APTT, s & $2.9 \pm 0.4$ & $1.7 \pm 0.2 *$ & $2.4 \pm 0.3$ & $4.7 \pm 0.2 *$ \\
\hline
\end{tabular}

Note: $*-p<0.05$ compared with the control group of patients 


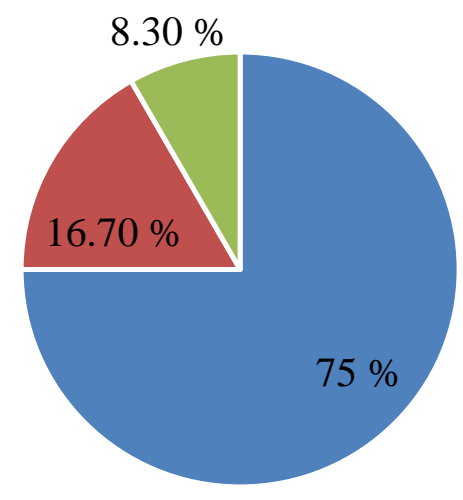

- Patients who received tranexamic acid between 3 and 8 hours

- Patients who received tranexamic acid in the prehospital stage

- Patients who received tranexamic acid on the first day $>8$ hours

Fig. 1. Frequency of administration of tranexamic acid in patients of the main group

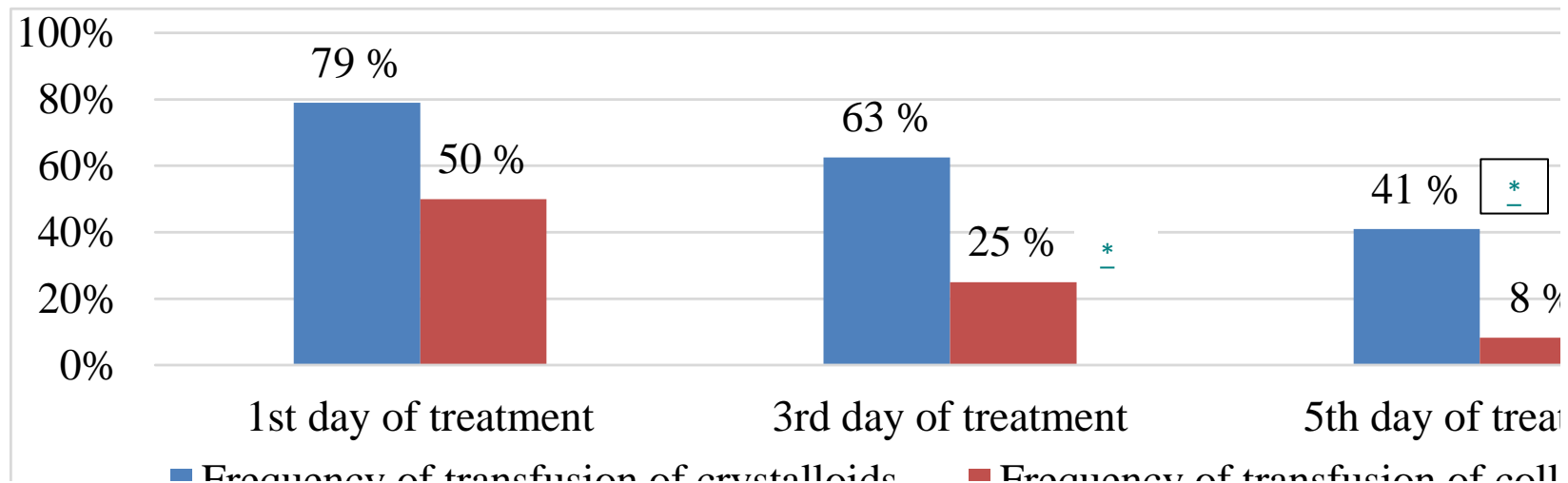

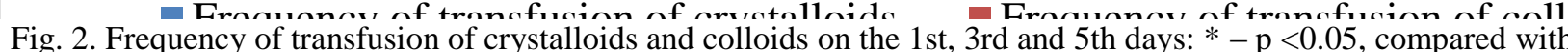
the previous stage of the treatment study

\section{Discussion of research results}

After analyzing the frequency of transfusion of erythrocyte mass and fresh-frozen plasma, it was found that transfusion throughout treatment was performed in 14 cases out of 24 , which was $58 \%$ of all cases of polytrauma. In 9 cases out of 24 - transfusion was performed on the first day, which is $37.5 \%$. The ratio of erythrocyte mass to fresh-frozen plasma volume on the first day of treatment was $1: 1$, erythrocyte mass was $475 \pm 35 \mathrm{ml}$, and fresh-frozen plasma volume was $458 \pm 15 \mathrm{ml}$.

Analyzing the literature, it should be noted that coagulopathy is one of the most common complications of polytrauma. The reason is a combination of shock caused by bleeding, tissue damage - associated with the regulation of thrombomodulin, the generation of thrombin-thrombomodulin complex, activation of anticoagulant and fibrinolytic systems [8]. Disorders of hemostasis after injury are associated not only with iatrogenic causes - impaired thrombin production and platelet function due to hypothermia, acidosis and dilution coagulopathy, and with modern hemostatic treatments dilution coagulopathy is rare. To induce clinical manifestations of traumainduced coagulopathy, the trigger is tissue damage (as a consequence, activation of the coagulation cascade, thrombin production and stimulation of anticoagulant pathways), which must be combined with tissue hypoperfusion (it is believed that this leads to contact, in- duced expression of thrombomodulin and endothelial protein $\mathrm{C}$ on the surface of the endothelium to activate protein $C$ ) [1]. The effect of trauma and shock on vascular platelet hemostasis remains unexplored [9], platelets in hemostasis in polytrauma play a critical role, and their low level predicts mortality [10]. Kornblith L. Z. et. al. demonstrated that the contribution of platelets to the strength of clots in trauma is higher than the contribution of fibrinogen. The main number of studies of primary hemostasis were performed using thromboelastography. Although the question of early platelet dysfunction in coagulopathy due to polytrauma remains unclear, a number of studies suggest that attenuation of platelet stimulation to adenosine diphosphate agonism may be secondary. Wohlauer M. V. et. al. [11] prospectively assessed platelet function in thrombus composition and stability, and found that platelet dysfunction is detected after serious injury and before significant fluid or blood injections.

Other studies have shown that in traumatized patients, platelets lose aggregative function as part of acute coagulopathy, which develops within minutes of injury, increases bleeding, and has a large impact on the risk of multiple organ failure and mortality. The decrease in the ability of platelets to aggregation occurs in parallel with an increase in their pro-coagulant function. It was found that circulating platelets turn into pro-coagulant balloons 
within a few minutes after injury, accompanied by the release of a large number of activated microparticles that cover the leukocytes [12]. In most patients with traumainduced coagulopathy, the number of platelets in the blood remains activated, but paradoxically impaired aggregation reactions ex vivo. This phenomenon is described as "platelet depletion" caused by trauma and shock [13].

Standard clinical parameters of the coagulation system do not provide sufficient information on platelet dysfunction in cases of post-traumatic coagulopathy and this makes post-traumatic coagulopathy a difficult diagnostic problem. Because platelet function in traumainduced coagulopathy has been underestimated for many years, current research should focus on studying the qualitative composition of platelets after injury. Disorders of platelet hemostasis can be assessed by determining the indicators of intravascular platelet activation. This study makes it possible to microscopically examine changes in platelet shapes, to determine the relationship between different platelet forms, to assess the nature and number of processes that formed on platelets in patients with polytrauma.

Primary hemostasis begins with reflex spasm of the arterioles, due to the release of platelets of catecholamines and serotonin. The next stage - adhesion - adhesion of platelets to unmasked collagen, their bonding with each other and the formation of aggregates - aggregation, as well as synthesis, accumulation and secretion by activating substances that stimulate adhesion and aggregation, and the formation of final platelet thrombus. Adhesion is facilitated by the unmasking of collagen due to damage to the vascular endothelium. Platelets begin to change their shape before contact with bare collagen. Initially, the shape of intact platelets changes from discoid (D) form to activated cells of disco echinocyte (DE), spherocytes (S) and, or, sphero echinocytes (SE). DE differs from D by the presence of single and short processes that appear after activation within the first second, and is the result of internal pressure on the plasma membrane of actin filaments. $\mathrm{C}$ is a more spherical cell, and SE is a spherical cell with a larger number of long processes. During the contact phase, the processes of activated platelets interact with the elements of the basement membrane of the vascular wall. The direct contact of platelet processes with collagen and the contact of platelets with collagen through Willebrand factor are important. An intact platelet has a discoid shape, however, such platelets have the ability to a limited number of reactions. They can interact with the collagen of damaged vessels and be included in the contact phase of adhesion to subendothelial structures. On surface D, under the influence of collagen, factor XI is activated without changing its shape. Thus $\mathrm{D}$, which is incapable of direct development of aggregation, secretion, refraction, can be subjected to receptor membrane activation and, accordingly, the altered forms are caused by substances for which there are specific receptors on the platelet membrane, i.e. thrombin, collagen, adenosine diphosphate (ADP), serotonin and other agonists. Platelet hemostatic activity occurs with the conversion of $\mathrm{D}$ to $\mathrm{DE}$, and $\mathrm{DE}$ is capable of both pronounced adhesion and aggregation, due to exposure in this phase on the plasma membrane of fibrinogen receptors. ADP, thromboxane A, biogenic amines - adrenaline, serotonin, platelet aggregation factor, thrombi are of the greatest importance for the emergence of the next stage - aggregation. Aggregates increase the permeability of the platelet membrane to calcium ions. Platelet aggregation is actually the connection of platelets to each other by bridges with ADP and calcium ions and plasma cofactors of aggregation. The appearance of appendages also promotes aggregation, increasing the likelihood of platelet collisions, which is necessary for this process. In the future, with the formation of a significant amount of SE, the aggregation activity decreases slightly and begins to develop refractoriness of the cell, which is most pronounced in SE. Understanding changes in the qualitative composition of platelets after injury is important to improve the treatment of coagulopathy, and studies show that platelet transfusions can increase morbidity and mortality after injury [1].

Study limitations. The presented fragment of the study aimed to examine whether the indicators of vascular-platelet and coagulation hemostasis in patients with trauma, compared with almost healthy individuals of the same age. In addition, the study was conducted on a very small sample size, which is insufficient to substantiate the role of vascular platelet hemostasis as a marker for post-traumatic coagulopathy.

Prospects for further research include continuing to study the indicators of vascular platelet hemostasis, which, accordingly, may lead to the development of new early diagnostic and therapeutic measures for the prevention and treatment of post-traumatic coagulopathy and its consequences.

\section{Conclusions}

1. Indicators of vascular-platelet hemostasis in patients with polytrauma had significant differences from those of the control group of patients at all stages of the study. It was found that on the first day the level of disco echinocytes was $8.2 \pm 1.1 \%$, on $\mathrm{d} 3-14.4 \pm 2.2 \%$, on $\mathrm{d} 5-12.1 \pm 2.4 \%,(\mathrm{p}<0.05)$, and the level of spherocytes at first was $1.5 \pm 0.1 \%$, at stage $\mathrm{d} 3-2.5 \pm 0.2 \%$, at stage $\mathrm{d} 5-3.1 \pm 0.4 \%(\mathrm{p}<0.05)$.

2. Indicators of coagulation hemostasis were normalized on the third day after the patient received polytrauma: in particular, the INR at d5 was $1.15 \pm 0.05$ ( $<<0.05$, compared with stage d1), APTT on day 5 reached $34.2 \pm 1.8 \mathrm{~s}(\mathrm{p}<0.05$, compared with step $\mathrm{d} 1)$.

3 . The indicators obtained by us may be evidence of a long and insufficiently controlled with the help of intensive care technologies of the process of activation of the vascular-platelet link system of hemostasis in polytrauma and indicate the prerequisites for late thrombotic complications among such patients.

\section{Conflicts of interest}

The authors declare that they have no conflicts of interest.

\section{Financing} port.
The study was performed without financial sup- 


\section{References}

1. Kornblith, L. Z., Moore, H. B., Cohen, M. J. (2019). Trauma- induced coagulopathy: The past, present, and future. Journal of Thrombosis and Haemostasis, 17 (6), 852-862. doi: http://doi.org/10.1111/jth.14450

2. Moore, E. E., Moore, H. B., Kornblith, L. Z., Neal, M. D., Hoffman, M., Mutch, N. J. et. al. (2021). Trauma-induced coagulopathy. Nature Reviews Disease Primers, 7 (1). doi: http://doi.org/10.1038/s41572-021-00264-3

3. Cardenas, J. C., Wade, C. E., Holcomb, J. B. (2014). Mechanisms of trauma-induced coagulopathy. Current Opinion in Hematology, 21 (5), 404-409. doi: http://doi.org/10.1097/moh.0000000000000063

4. Solomon, C., Traintinger, S., Ziegler, B., Hanke, A., Rahe-Meyer, N., Voelckel, W., Schöchl, H. (2011). Platelet function following trauma. Thrombosis and Haemostasis, 106 (8), 322-330. doi: http://doi.org/10.1160/th11-03-0175

5. Kornblith, L. Z., Robles, A. J., Conroy, A. S., Hendrickson, C. M., Calfee, C. S., Fields, A. T. et. al. (2018). Perhaps it's not the platelet: ristocetin uncovers the potential role of von Willebrand factor in impaired platelet aggregation following traumatic brain injury. Journal of Trauma and Acute Care Surgery, 85 (5), 873-880. doi: http://doi.org/10.1097/ta.0000000000002025

6. Sirajuddin, S., Valdez, C., DePalma, L., Maluso, P., Singhal, R., Schroeder, M., Sarani, B. (2016). Inhibition of platelet function is common following even minor injury. Journal of Trauma and Acute Care Surgery, 81 (2), 328-332. doi: http://doi.org/10.1097/ ta.0000000000001057

7. ISTH Criteria for Disseminated Intravascular Coagulation. Available at: https://www.mdcalc.com/isth-criteriadisseminated-intravascular-coagulation-dic\#why-use

8. Hagemo, J. S., Stanworth, S., Juffermans, N. P., Brohi, K., Cohen, M., Johansson, P. I. et. al. (2014). Prevalence, predictors and outcome of hypofibrinogenaemia in trauma: a multicentre observational study. Critical Care, 18 (2), R52. doi: http://doi.org/10.1186/ cc13798

9. Kornblith, L. Z., Kutcher, M. E., Redick, B. J., Calfee, C. S., Vilardi, R. F., Cohen, M. J. (2014). Fibrinogen and platelet contributions to clot formation: implications for trauma resuscitation and thromboprophylaxis. Journal of Trauma and Acute Care Surgery, 76 (2), 255-263. doi: http://doi.org/10.1097/ta.0000000000000108

10. Brown, L. M., Call, M. S., Margaret Knudson, M., Cohen, M. J. (2011). A Normal Platelet Count May Not Be Enough: The Impact of Admission Platelet Count on Mortality and Transfusion in Severely Injured Trauma Patients. Journal of Trauma: Injury, Infection \& Critical Care, 71 (2), S337-S342. doi: http://doi.org/10.1097/ta.0b013e318227f67c

11. Wohlauer, M. V., Moore, E. E., Thomas, S., Sauaia, A., Evans, E., Harr, J. et. al. (2012). Early Platelet Dysfunction: An Unrecognized Role in the Acute Coagulopathy of Trauma. Journal of the American College of Surgeons, 214 (5), 739-746. doi: http://doi.org/10.1016/j.jamcollsurg.2012.01.050

12. Napolitano, L. M., Cohen, M. J., Cotton, B. A., Schreiber, M. A., Moore, E. E. (2013). Tranexamic acid in trauma: how should we use it. Journal of Trauma and Acute Care Surgery, 74 (6), 1575-1586. doi: http://doi.org/10.1097/ta.0b013e318292cc54

13. Plautz, W. E., Matthay, Z. A., Rollins- Raval, M. A., Raval, J. S., Kornblith, L. Z., Neal, M. D. (2020). Von Willebrand factor as a thrombotic and inflammatory mediator in critical illness. Transfusion, 60 (S3), S158-S166. doi: http://doi.org/10.1111/trf.15667

Received date 06.05.2021

Accepted date 14.06.2021

Published date 30.07.2021

Mariana Vyshynska, Assistant, Department of Anesthesiology and Intensive Care, Danylo Halytskyi Lviv National Medical University, Pekarska str., 69, Lviv, Ukraine, 79010

E-mail: mariana.vyshynska@gmail.com 